\title{
Admissible Clustering of Aggregator Components: A Necessary and Sufficient Stochastic Semi-Nonparametric Test for Weak Separability
}

\author{
William A. Barnett ${ }^{1}$ \\ Philippe de Peretti ${ }^{2}$ \\ University of Kansas Université de la Sorbonne
}

November 3, 2008

\footnotetext{
${ }^{1}$ Department of Economics, University of Kansas, Snow Hall, 1460 Jayhawk Blvd., Room 356, Lawrence, KS 66045-7585. Phone: 785-864-2844. Fax: 785-864-5760. Email: barnett@ku.edu

${ }^{2}$ Centre d'Economie de la Sorbonne. Maison des Sciences Economiques, 106-112 boulevard de l'Hôpital 75013 Paris, France. Phone: 00331440782 69, email: philippe.de-peretti@univ-paris1.fr
} 


\begin{abstract}
In aggregation theory, the admissibility condition for clustering together components to be aggregated is blockwise weak separability, which also is the condition needed to separate out sectors of the economy. Although weak separability is thereby of central importance in aggregation and index number theory and in econometrics, prior attempts to produce statistical tests of weak separability have performed poorly in Monte Carlo studies. This paper deals with seminonparametric tests for weak separability. It introduces both a necessary and sufficient test, and a fully stochastic procedure allowing to take into account measurement error. Simulations show that the test performs well, even for large measurement errors.
\end{abstract}




\section{Introduction}

This paper deals with semi-nonparametric testing procedures for models of the form:

$$
U\left(\mathbf{x}_{i}^{*}\right)=V\left(\mathbf{x}_{i}^{*(2)}, f\left(\mathbf{x}_{i}^{*(1)}\right)\right), i=1, \ldots, T
$$

where:

$U()$ is a utility function,

$V()$ is a macro function,

$f()$ is a micro function,

$\mathbf{x}_{i}^{*}$ is a vector of real commodities,

$\mathbf{x}_{i}^{*(1)}$ and $\mathbf{x}_{i}^{*(2)}$ are two partitions of $\mathbf{x}_{i}^{*}$ such that $\mathbf{x}_{i}^{*(1)} \cup \mathbf{x}_{i}^{*(2)}=\mathbf{x}_{i}^{*}$ and $\mathbf{x}_{i}^{*(1)} \cap$ $\mathbf{x}_{i}^{*(2)}=\varnothing$.

Moreover, the tests considered here also deal with the common situation when $\mathbf{x}_{i}^{*}$ is not directly observed by the econometrician. Only $\mathbf{x}_{i}$ is observed, related to $\mathbf{x}_{i}^{*}$ by $(2)$.

$$
\mathbf{x}_{i}=g\left(\mathbf{p}_{i}\right)+\boldsymbol{\psi}_{i}
$$

where:

$\mathbf{x}_{i}^{*}=g\left(\mathbf{p}_{i}\right), g\left(\mathbf{p}_{i}\right)$ is unknown,

$\boldsymbol{\psi}_{i}$ is a vector of zero mean iid terms with unknown diagonal covariance matrix, $\mathbf{p}_{i}$ is a vector of prices.

Model (1) has been extensively studied, especially within the revealed preference framework. Varian (1983) has first proposed a fully nonparametric procedure based on the well-known Generalized Axiom of Revealed Preference (GARP). Among others, Swofford and Whitney (1987) have implemented such an approach. Nevertheless, Barnett and Choi (1989) have cast some doubts on the validity of the procedure. On simulated data they have showed that the test was strongly biased toward rejection. Two factors are generally admitted to explain this high rejection rate: i) The test is non-stochastic. Being constructed as a three-step test of utility maximization, it uses each step the purely deterministic GARP, therefore totally ignoring model (2). Thus, a single violation of the axiom leads to reject the null of weak separability, even if caused by purely 
stochastic causes as measurement error. ii) The step three of the procedure requires utility and price indices for the sub-utility to be computed by solving the so-called Afriat inequalities. Nevertheless, as showed by Fleissig and Whitney (2003), the way the inequalities are solved dramatically influences the power of the test. Moreover, this leads to an only sufficient condition.

Three approaches have tried to correct the initial nonparametric approach. Fleissig and Whitney (2003) have suggested a new algorithm to solve the Afriat inequalities. They have moreover showed that their test performed well, even if data were measured with small errors. Jones et al. (2005), based on Varian (1985) and Swofford and Whitney (1994) have introduced a modified weak separability test that explicitly deals with (2) and incomplete adjustment models. They have also suggested a necessary and sufficient test. At last, based on de Peretti (2005), de Peretti (2007) has also introduced a stochastic weak separability test. He has moreover suggested a necessary and sufficient test under homotheticity, or uses the new algorithm of Fleissig and Whitney (2003) under the more general case.

Nevertheless, these three approaches are not totally satisfactory. The first approach clearly remains non-stochastic. It does not explicitly deal with measurement error, i.e. not allowing to test the significance of the violations of $\mathrm{GARP}^{1}$. The second one is extremely computationally burdensome, preventing its use for large datasets. Moreover, it uses the Varian's (1985) lower bound test, leading to a quite unclear decision rule. At last, even if the de Peretti's (2007) approach allows to test the significance of the deviation from weak separability, it remains within the Afriat inequalities framework under the general non-homothetic case. It then produces an only sufficient condition.

The goal of this paper is to introduce a new class of weak separability tests. With regard to the above works, the test we want to consider differs in two ways. First it is semi-nonparametric in the sense that it uses nonparametric tests to check the maximization assumptions, but parametric ones to test the weak separability condition. Nevertheless, it should be noted that the procedure remains parametric flexible form free, in the sense that no estimation of 
$g\left(\mathbf{p}_{i}\right)$ is required. Moreover, the test is defined beyond the Afriat inequalities framework and produces a necessary and sufficient condition. Second, the whole procedure is fully stochastic. Based on model (2), the violations of GARP are tested for their significance. Also, the separability condition we use allows for measurement error or small optimization errors. Results from a small Monte Monte Carlo simulations show that the procedure performs well, even for large measurement errors.

This paper is structured as follows. Standard deterministic nonparametric tests for separability are discussed in Section 2. In Section 3, we introduce an alternative test for weak separability, replacing the standard Afriat inequalities based condition by a necessary and sufficient one. Section 4 extends the approach to deal with measurement error. In Section 5, we perform a small Monte Carlo simulations to analyze the power of the test. At last, Section 6 concludes.

\section{Preliminaries}

We first assume that $\boldsymbol{\psi}_{i}=\mathbf{0}, i=1, \ldots, T$, meaning that the data are perfectly observed. Let $\mathbf{X}\left(=\mathbf{X}^{*}\right)$ be a $(T \times k)$ matrix of observed real quantities, where $T$ denotes the number of observations and $k$ the number of goods. Let $\mathbf{x}_{i}=$ $\left(x_{i 1}, x_{i 2}, \ldots, x_{i k}\right)^{\prime}$ be the $i$ th row of the matrix, $i=1, \ldots, T$. Similarly, define $\mathbf{P}$ as a $(T \times k)$ matrix of corresponding prices, and let $\mathbf{p}_{i}=\left(p_{i 1}, p_{i 2}, \ldots, p_{i k}\right)^{\prime}$ be the $i$ th row of the matrix, $i=1, \ldots, T$. Now consider two partitions of $\mathbf{X}$, the $(T \times a) \mathbf{X}^{(1)}$ matrix, $a \in\{1, \ldots, k-1\}$, with $\mathbf{x}_{i}^{(1)}=\left(x_{i 1}, x_{i 2}, \ldots, x_{i a}\right)^{\prime}$, and the $(T \times(k-a)) \mathbf{X}^{(2)}$ matrix with $\mathbf{x}_{i}^{(2)}=\left(x_{i(a+1)}, x_{i(a+2)}, \ldots, x_{i k}\right)^{\prime}$. Let $\mathbf{P}^{(1)}$ and $\mathbf{P}^{(2)}$ be the corresponding associated price matrices, with $\mathbf{p}_{i}^{(1)}=\left(p_{i 1}, p_{i 2}, \ldots, p_{i a}\right)^{\prime}$ and $\mathbf{p}_{i}^{(2)}=\left(p_{i(a+1)}, p_{i(a+2)}, \ldots, p_{i k}\right)^{\prime}$. Define the weak separability of the first $a$ columns of $\mathbf{X}$, that is of $\mathbf{X}^{(1)}$, as follows:

Definition 1: There is weak separability if there exists a utility function (3) 
rationalizing the data, and if this latter admits a rewriting (4).

$$
\begin{aligned}
U_{i} & =U\left(\mathbf{x}_{i}\right) \\
U_{i} & =V\left(\mathbf{x}_{i}^{(2)}, f\left(\mathbf{x}_{i}^{(1)}\right)\right), i=1, \ldots, T
\end{aligned}
$$

where:

$U()$ is the overall utility function,

$V()$ is a strictly increasing function, known as the macro-function,

$f()$ is the sub-utility function, or the micro-function. It is also the aggregator function if homothetic.

Following Deaton and Muellbauer (1980), note that the weak separability implies that the marginal rate of substitution between any two goods of the separable group is independent of the goods outside the group, i.e.:

$$
\partial\left(\frac{\frac{\partial U\left(\mathbf{x}_{i}\right)}{\partial x_{i j}}}{\frac{\partial U\left(\mathbf{x}_{i}\right)}{\partial x_{i l}}}\right) / \partial x_{i m}=0, j, l=1, \ldots, a ; j \neq l ; m=a+1, \ldots, k
$$

Varian (1983), based on Varian (1982), has developed a procedure in order to test for weak separability that ignores the above condition (5). In order to meet the weak separability criterion, three conditions must be fulfilled, that is $U(), f()$ and $V()$ must exist. Testing for weak separability therefore reduces to a three-step test of utility maximization. Each step uses the well-known Generalized Axiom of Revealed Preference (GARP), introduced hereafter.

Define the three following binary relations: $\mathbf{x}_{i}$ is said to be strictly directly revealed preferred to $\mathbf{x}_{j}$ if $\mathbf{p}_{i} \cdot \mathbf{x}_{i}>\mathbf{p}_{i} \cdot \mathbf{x}_{j}$, written $\mathbf{x}_{i} P^{0} \mathbf{x}_{j} ; \mathbf{x}_{i}$ is said to be directly revealed preferred to $\mathbf{x}_{j}$ if $\mathbf{p}_{i} \cdot \mathbf{x}_{i} \geq \mathbf{p}_{i} \cdot \mathbf{x}_{j}$, written $\mathbf{x}_{i} R^{0} \mathbf{x}_{j}$; at last, $\mathbf{x}_{i}$ is said to be revealed preferred to $\mathbf{x}_{j}$ if $\mathbf{x}_{i} R^{0} \mathbf{x}_{m}, \mathbf{x}_{m} R^{0} \mathbf{x}_{k}, \ldots, \mathbf{x}_{p} R^{0} \mathbf{x}_{j}$, written $\mathbf{x}_{i} R \mathbf{x}_{j}$, where $R$ is the transitive closure of $R^{0}$. Using the above definitions, GARP is defined as follows:

Definition 2 (GARP): For a couple of observations $(i, j) i \in\{1, \ldots, T\}, j \in$ $\{1, \ldots, T\}: \mathbf{x}_{i} R \mathbf{x}_{j} \Longrightarrow \mathbf{p}_{j} \cdot \mathbf{x}_{j} \leq \mathbf{p}_{j} \cdot \mathbf{x}_{i}$ (or $\mathbf{x}_{i} R \mathbf{x}_{j}$ implies not $\mathbf{p}_{j} \cdot \mathbf{x}_{j}>\mathbf{p}_{j} \cdot \mathbf{x}_{i}$ ). 
Using GARP, Varian (1982) proved that:

Theorem 1 (Varian 1982): For a set $\left\{\mathbf{x}_{i}, \mathbf{p}_{i}\right\}_{i=1}^{T}$, the three following conditions are equivalent:

i) There exists a locally non-satiated utility function $U()$ that rationalizes the data,

ii) There exist strictly positive utility indices $U_{i}$ and marginal income indices $\lambda_{i}$ that satisfy $\forall i \in\{1, \ldots, T\} \forall j \in\{1, \ldots, T\}$ the Afriat inequalities (6),

$$
U_{i} \leqslant U_{j}+\lambda_{j}\left(\mathbf{p}_{j} \cdot \mathbf{x}_{i}-\mathbf{p}_{j} \cdot \mathbf{x}_{j}\right)
$$

iii) The data satisfy GARP.

Testing for the weak separability of $\mathbf{X}^{(1)}$ is now straightforward. It amounts to checking if the three following conditions hold:

Condition 1: GARP holds for $\left\{\left(\mathbf{x}_{i}, \mathbf{p}_{i}\right)\right\}_{i=1}^{T}$, that is $U()$ exists.

Condition 2: GARP holds for $\left\{\left(\mathbf{x}_{i}^{(1)}, \mathbf{p}_{i}^{(1)}\right)\right\}_{i=1}^{T}$, that is $f()$ exists.

Condition 3: GARP holds for $\left\{\left(\left(\mathbf{x}_{i}^{(2)}, U_{i}\right),\left(\mathbf{p}_{i}^{(2)}, \lambda_{i}^{-1}\right)\right)\right\}_{i=1}^{T}$, where $U_{i}$ and $\lambda_{i}$ are strictly positive indices satisfying (6) for $\left\{\mathbf{x}_{i}^{(1)}, \mathbf{p}_{i}^{(1)}\right\}_{i=1}^{T}$, that is $\mathbf{X}^{(1)}$ is weakly separable in $U()$.

One will find in Swofford and Whitney (1987) or in Fisher and Fleissig (1997) implementations of such an approach.

There are clearly two major drawbacks with the above test. First, the above condition 3 is an only sufficient one. Thus, if it does not hold, one can not be sure that for an other set $\left\{U_{i}, \lambda_{i}\right\}_{i=1}^{T}$, GARP won't be violated. Moreover the power of the test dramatically depends on the way the indices are computed (see Fleissig and Whitney 2003). Second, the whole procedure is clearly nonstochastic, and a single violation of GARP leads to reject the null, even if caused by measurement error, or other purely stochastic causes. We first deal with the first point, by introducing a necessary and sufficient alternative condition 3 , and then introduce a stochastic extension. 


\section{$3 \quad$ An alternative condition 3}

To introduce the new test for weak separability, assume that the conditions 1 and 2 hold, that is the two sets $\left\{\mathbf{x}_{i}, \mathbf{p}_{i}\right\}_{i=1}^{T}$ and $\left\{\mathbf{x}_{i}^{(1)}, \mathbf{p}_{i}^{(1)}\right\}_{i=1}^{T}$ are consistent with GARP. What we want, is to replace the above condition 3 by a necessary and sufficient one. The one we want to consider is based on (5), that is on the independence between the marginal rate of substitution between any two goods of the separable group and the goods outside the group.

The difficulty with such an approach is that the form of the sub-utility, and then of course the gradient matrix, are unknown. Nevertheless, even if unknown, it is possible to directly compute the ratio of the first order derivatives at first order conditions. Indeed, if GARP holds for the set $\left\{\left(\mathbf{x}_{i}^{(1)}, \mathbf{p}_{i}^{(1)}\right)\right\}_{i=1}^{T}$ then, given theorem (1) exists a sub-utility $f\left(\mathbf{x}_{i}^{(1)}\right)$ rationalizing the data. Therefore the following lemma can be applied:

Lemma 1 (Konyus and Byushgens): Suppose $f()$ is differentiable, and that GARP holds for $\left\{\left(\mathbf{x}_{i}^{(1)}, \mathbf{p}_{i}^{(1)}\right)\right\}_{i=1}^{T}$, that is each $\mathbf{x}_{i}^{(1)}$ is a solution of the program $\max _{x}\left\{f(\mathbf{x}): \mathbf{p}_{i}^{(1)} \cdot \mathbf{x} \leq \mathbf{p}_{i}^{(1)} \cdot \mathbf{x}_{i}^{(1)}, \mathbf{x} \geq \mathbf{0}\right\}$ then:

$$
\frac{\mathbf{p}_{i}^{(1)^{\prime}}}{\mathbf{p}_{i}^{(1)} \cdot \mathbf{x}_{i}^{(1)}}=\frac{\nabla f\left(\mathbf{x}_{i}^{(1)^{\prime}}\right)}{\mathbf{x}_{i}^{(1)} \cdot \nabla f\left(\mathbf{x}_{i}^{(1)}\right)}
$$

Dividing the $j$ th row of the system (7) by the $l$ th row $j, l=1, \ldots, a, j \neq l$, returns the well-known condition:

$$
\frac{p_{i j}^{(1)}}{p_{i l}^{(1)}}=\frac{\frac{\partial f\left(\mathbf{x}_{i}^{(1)}\right)}{\partial x_{i j}^{(1)}}}{\frac{\partial f\left(\mathbf{x}_{i}^{(1)}\right)}{\partial x_{i l}^{(1)}}}
$$

At first order conditions, the marginal rate of substitution between two goods equals the corresponding price ratio. Thus, in order to test for weak separability, knowing the form of the first-order derivatives of the sub-utility is not necessary, since we are able to directly compute the output of the marginal rates of substitution. Therefore, testing for weak separability amounts to checking the independence between all the unique price ratios of the goods inside the possibly separable group, and the quantities outside the group. Let $\mathbf{Y}$ be a 
$\left(T \times \sum_{i=1}^{a-1}(a-i)\right)$ matrix $^{2}$ of the corresponding price ratios of the separable group, defined as:

$$
\mathbf{Y}^{\prime}=\left[\begin{array}{c}
\left(\log \left(\frac{\mathbf{p} \cdot 1}{\mathbf{p}_{\cdot 2}}\right)\right)^{\prime} \\
\vdots \\
\left(\log \left(\frac{\mathbf{p}_{\cdot 1}}{\mathbf{p}_{\cdot}}\right)\right)^{\prime} \\
\left(\log \left(\frac{\mathbf{p} \cdot 2}{\mathbf{p}_{.3}}\right)\right)^{\prime} \\
\vdots \\
\left(\log \left(\frac{\mathbf{p} \cdot 2}{\mathbf{p}_{\cdot a}}\right)\right)^{\prime} \\
\vdots \\
\left(\log \left(\frac{\mathbf{p}_{\cdot(a-1)}}{\mathbf{p}_{\cdot a}}\right)\right)^{\prime}
\end{array}\right]
$$

where:

$\mathbf{p}_{\cdot j}=\left(p_{1 j}, p_{2 j}, \ldots, p_{T j}\right)$ is the $j$ th column of $\mathbf{P}^{(1)}, j=1, \ldots, a$.

Define the model (9) that can be re-written as (10).

$$
\begin{aligned}
\mathbf{Y} & =\mathbf{X}^{(3)} \boldsymbol{\beta}+\mathbf{E} \\
\mathbf{Y} & =\left[\mathbf{1} \log \left(\mathbf{X}^{(1)}\right) \log \left(\mathbf{X}^{(2)}\right)\right]\left[\begin{array}{l}
\boldsymbol{\beta}^{(1)} \\
\boldsymbol{\beta}^{(2)}
\end{array}\right]+\mathbf{E}
\end{aligned}
$$

where:

$\mathbf{Y}$ is a $\left(T \times \Sigma_{i=1}^{a-1}(a-i)\right)$ matrix,

$\mathbf{X}^{(3)}$ is a $(T \times(k+1))$ matrix defined as $\mathbf{X}^{(3)}=[\mathbf{1} \log (\mathbf{X})]$,

$\boldsymbol{\beta}$ is a $\left((k+1) \times \sum_{i=1}^{a-1}(a-i)\right)$ of parameters,

$\mathbf{E}$ is a $\left(T \times \Sigma_{i=1}^{a-1}(a-i)\right)$ matrix of residuals,

$\boldsymbol{\beta}^{(1)}$ is a $\left((a+1) \times \Sigma_{i=1}^{a-1}(a-i)\right)$ matrix of parameters,

$\boldsymbol{\beta}^{(2)}$ is a $\left((k-a) \times \Sigma_{i=1}^{a-1}(a-i)\right)$ matrix of parameters.

Then, clearly weak separability of the first $a$ rows of $\mathbf{X}$ implies the nullity of the coefficients matrix $\boldsymbol{\beta}^{(2)}$. Therefore, testing for the weak separability of $\mathbf{X}^{(1)}$ amounts to checking if the three following conditions hold:

Condition 4: GARP holds for $\left\{\left(\mathbf{x}_{i}, \mathbf{p}_{i}\right)\right\}_{i=1}^{T}$, that is $U()$ exists. 
Condition 5: GARP holds for $\left\{\left(\mathbf{x}_{i}^{(1)}, \mathbf{p}_{i}^{(1)}\right)\right\}_{i=1}^{T}$, that is $f()$ exists.

Condition 6: $\boldsymbol{\beta}^{(2)}=\mathbf{0}$ in (9), that is $\mathbf{X}^{(1)}$ is weakly separable in $U()$.

We now turn to a stochastic extension of the procedure ${ }^{3}$.

\section{A stochastic extension}

We now relax the unrealistic assumption that data are perfectly observed and assume that $\boldsymbol{\psi}_{i} \neq \mathbf{0}, i=1, \ldots, T$.

Assumption 1: Under the null, $\mathbf{X}^{*}$ is generated by a weakly separable utility function, but is unobservable. Only $\mathbf{X}$ is observed. It relates to $\mathbf{X}^{*}$ by the additive relation:

$$
x_{i j}=x_{i j}^{*}+\psi_{i j}
$$

Assumption 2: The terms $\psi_{i j}$ are iid with zero mean and variance $\sigma_{\psi_{j}}^{2}$, with distribution function $F_{j}(x)$. The distribution $F_{j}(x)$ is max and min-stable.

Equation (11) has two major implications. First, it leads to take into account that some violations of GARP when testing for conditions 1 and 2, might be caused by purely stochastic factors. Hence the need for discriminating between significant and non significant violations of the axiom. Second, if the violations are non-significant, and thus if the data are measured with errors, model (9) is to be estimated by using particular estimators. We first focus on the way to test the significance of the violations of GARP when testing for weak separability.

The procedure we use is an extension of de Peretti $(2005,2007)$. It therefore inherits a similar logical structure, consisting in:

i) Finding the minimal adjustment in order for the data to be consistent with both conditions 1 and 2,

ii) Testing the significance of this adjustment.

Concerning the former, computing the minimal adjustment is achieved by 
solving over $z_{i j}$ the quadratic program:

$$
o b j=\min _{z_{i j}} \sum_{i=1}^{T} \sum_{j=1}^{k}\left(x_{i j}-z_{i j}\right)^{2}
$$

Subject to :

$$
\begin{aligned}
& \mid \forall i \epsilon\{1, \ldots, T\} \forall j \epsilon\{1, \ldots, T\}: \mathbf{z}_{i} R \mathbf{z}_{j} \Longrightarrow \mathbf{p}_{j} \cdot \mathbf{z}_{j} \leq \mathbf{p}_{j} \cdot \mathbf{z}_{i} \\
& \mid \begin{array}{l}
\forall i \epsilon\{1, \ldots, T\} \forall j \epsilon\{1, \ldots, T\}: \mathbf{z}_{i}^{(1)} R \mathbf{z}_{j}^{(1)} \Longrightarrow \mathbf{p}_{j}^{(1)} \cdot \mathbf{z}_{j}^{(1)} \leq \mathbf{p}_{j}^{(1)} \cdot \mathbf{z}_{i}^{(1)} \\
\text { where: } \\
\mathbf{z}_{i}^{(1)}=\left(z_{i 1}, z_{i 2}, \ldots, z_{i a}\right) \text { and } \mathbf{p}_{i}^{(1)}=\left(p_{i 1}, p_{i 2}, \ldots, p_{i a}\right)
\end{array}
\end{aligned}
$$

Let $\widehat{\mathbf{Z}}$ be the matrix solution of the above program and define $\widehat{\mathbf{\Omega}}=\mathbf{X}-\widehat{\mathbf{Z}}$ as the matrix of residuals. $\widehat{\Omega}$ is the minimal adjustment in the data in order for $\widehat{\mathbf{Z}}$ to satisfy both condition 1 (constraint C.1) and condition 2 (constraint C.2). We will therefore refer to these residuals as theoretical residuals ${ }^{4}$.

To test the significance of the adjustment, that is to test whether or not violations are due to measurement error, we compare the theoretical residuals with the true measurement error $\Psi=\mathbf{X}-\mathbf{X}^{*}$. For convenience, we will first assume that $\Psi$ is known as well its distribution function. Since in (12) only few bundles are adjusted, the idea is to build a test tracking excess adjustments in some goods, and in particular to test whether or not extremes theoretical residuals are consistent with the perturbations induced by measurement error $^{5}$. Statistically, we proceed as follows. For one good $j, j=1, \ldots, k$ define $\widehat{\operatorname{Max}}_{j}=\max \left(\widehat{\omega}_{1 j}, \widehat{\omega}_{2 j}, \ldots, \widehat{\omega}_{T j}\right)$ and $\widehat{\operatorname{Min}}_{j}=\min \left(\widehat{\omega}_{1 j}, \widehat{\omega}_{2 j}, \ldots, \widehat{\omega}_{T j}\right)$, where $\left(\widehat{\omega}_{1 j}, \widehat{\omega}_{2 j}, \ldots, \widehat{\omega}_{T j}\right)$ is the $j$ th column of the $(T \times k)$ matrix $\widehat{\boldsymbol{\Omega}}$. Similarly define $\operatorname{Max}_{j}=\max \left(\psi_{1 j}, \psi_{2 j}, \ldots, \psi_{T j}\right)$ and $\operatorname{Min}_{j}=\min \left(\psi_{1 j}, \psi_{2 j}, \ldots, \psi_{T j}\right)$. Since $\operatorname{Min}_{j}=\min \left(\psi_{1 j}, \psi_{2 j}, \ldots, \psi_{T j}\right)=-\max \left(-\psi_{1 j},-\psi_{2 j}, \ldots,-\psi_{T j}\right)$, and since reversal of the sign of $\left(\psi_{1 j}, \psi_{2 j}, \ldots, \psi_{T j}\right)$ will produce results for the smallest extreme, we will only focus on theoretical results concerning the largest extreme. Then, under Assumption 2, there exists a set of constants $a_{T j} \in \mathbb{R}$ and $b_{T j}>0$ and the following theorem holds:

Theorem 2 (Fisher-Tippett): For the iid sequence $\left(\psi_{1 j}, \psi_{2 j}, \ldots, \psi_{T j}\right)$, if 
there exists norming constants $a_{T j} \in R$ and $b_{T j}>0$ and some non-degenerate distribution function $G$ such that:

$$
\left(\operatorname{Max}_{j}-a_{T j}\right) b_{T j}^{-1} \stackrel{\mathcal{L}}{\rightarrow} G
$$

Then $G$ belongs to the type of one of the following three laws:

$$
\begin{aligned}
& \text { Fréchet (type III) : } \quad G_{3}(x)=\left\{\begin{array}{ll}
0, & x \leq 0 \\
\exp \left(-x^{-\alpha_{j}}\right), & x>0
\end{array} \alpha_{j}>0 .\right. \\
& \text { Weibull (type II) : } G_{2}(x)=\left\{\begin{array}{ll}
\exp \left(-\left(-x^{\alpha_{j}}\right)\right), & x \leq 0 \\
1, & x>0
\end{array} \alpha_{j}>0 .\right. \\
& \text { Gumbel (type I) : } G_{1}(x)=\exp (-\exp (-x)), \quad x \in \mathbb{R} \text {. }
\end{aligned}
$$

With a prior knowledge of the true distribution of the errors, it is possible to know the domain of attraction of the law of the extremes, and then choosing (14), (15), or (16). Note that a post validation is always possible and desirable using for instance probability plots or goodness-of-fit measures (see D'Agostino and Stephens 1986). Under the general normality assumption of the true measurement error, which is our framework here, it can be shown that the two extremes belong to the domain of attraction of the type I Gumbel law. Therefore, under this assumption, testing the significance of the adjustments for good $j$, for non-centered and non-reduced variables, is achieved by computing the two p-values:

$1-P[X \leq x]=1-G_{1}(x)=1-\exp \left(-\exp \left(-\frac{\left(x-\mu_{j}\right)}{\sigma_{j}}\right)\right)$, where $x=\widehat{\operatorname{Max}}_{j}$

for the maxima, and

$$
1-P[X \leq x]=1-G_{1}(x)=1-\exp \left(-\exp \left(\frac{\left(x-\mu_{j}\right)}{\sigma_{j}}\right)\right), \text { where } x=\widehat{\operatorname{Min}}_{j}
$$

for the minima.

where $\mu_{j}$ is the location parameter, and $\sigma_{j}$ the scale parameter, computed using the distribution of $\boldsymbol{\Psi}$. 
The two tests simply return the probability for the two extremes of the theoretical residuals to belong to the distribution of the extremes of the true measurement error. Hence, excess adjustments will lead to a rejection of the null.

With no prior knowledge, the three laws can be expressed as the Generalized Extreme Value distribution (GEV), also known as the Von Mises-Jenkinson type distribution (19). See Hosking, Wallis and Wood (1985) and Hosking (1985) for estimation methods for the parameters.

$$
G(x)= \begin{cases}\exp \left(-\left(1+\lambda \frac{\left(x-\mu_{j}\right)}{\sigma_{j}}\right)^{-\frac{1}{\lambda}}\right), & 1+\lambda \frac{\left(x-\mu_{j}\right)}{\sigma_{j}}>0, \lambda>0, \\ \exp \left(-\exp \left(-\frac{\left(x-\mu_{j}\right)}{\sigma_{j}}\right)\right), & \lambda=0 .\end{cases}
$$

Empirically, the realization $\boldsymbol{\Psi}$ is seldom observable. We thus need an estimate $\widehat{\boldsymbol{\Psi}}$ of $\boldsymbol{\Psi}$, as well as several realizations of the extremes in order to compute the parameters $\mu_{j}$ and $\sigma_{j}, j=1, \ldots, k$. Interestingly, (11) can be seen as the measurement equation of a time-invariant state space model. Indeed, assuming a first order Markov transition process for the unobservable state variable, returns the model (20) for good $j$.

$$
\begin{aligned}
x_{i j} & =z_{i j}+\psi_{i j} \\
z_{(i+1) j} & =F z_{i j}+a_{j}+\xi_{i j}
\end{aligned}
$$

where:

$z_{i j}$ is the estimated unobserved quantity,

$\psi_{i j}$ and $\xi_{i j}$ are two uncorrelated residuals, respectively with variance $\sigma_{\psi_{j}}^{2}$ and $\sigma_{\xi_{j}}^{2}$,

$F, a_{j}, \sigma_{\psi_{j}}^{2}$ and $\sigma_{\xi_{j}}^{2}$ are the hyperparameters of the model. $F$ being assumed to be unity in most applications.

One will find in Harvey (1989) or more recently in Reinsel (1997) or Durbin and Koopman (2001) comprehensive studies of such models. Define $z_{i j \mid T}$ as the smoothed estimates of the state space vector obtained via the Kalman filter ${ }^{6}$, and then $\widehat{\boldsymbol{\psi}}_{j}=\left(\widehat{\psi}_{1 j}, \widehat{\psi}_{2 j}, \ldots, \widehat{\psi}_{T j}\right)=\left(x_{1 j}-z_{1 j \mid T}, x_{2 j}-z_{2 j \mid T}, . ., x_{T j}-z_{T j \mid T}\right)$ 
and $\widehat{\operatorname{Max}}_{j}=\max \left(\widehat{\psi}_{1 j}, \widehat{\psi}_{2 j}, \ldots, \widehat{\psi}_{T j}\right)$ and $\widehat{\operatorname{Min} \psi}_{j}=\min \left(\widehat{\psi}_{1 j}, \widehat{\psi}_{2 j}, \ldots, \widehat{\psi}_{T j}\right)$. In order to have several realizations of the two extremes to compute the location and scale parameters, we perform a parametric bootstrap. Following Stoffer and Wall (1991) define for good $j, z_{(i+1) j \mid i}$ as the best linear linear predictor of $z_{(i+1) j}$ computed thanks to the Kalman filter. Putting the Kalman filter into the innovation representation form returns equations (21) and (22).

$$
\begin{aligned}
z_{(i+1) j \mid i} & =F z_{i j \mid i-1}+F K_{i j} \epsilon_{i j} \\
x_{i j} & =z_{i j \mid i-1}+\epsilon_{i j}
\end{aligned}
$$

where :

$\epsilon_{i j}=x_{i j}-z_{i j \mid i-1}$, are the innovations,

$K_{i j}=P_{i j \mid i-1} \Sigma_{i j}^{-1}$ is the Kalman gain, with $P_{i j \mid i-1}$ the covariance matrix of $z_{i j}-z_{i j \mid i-1}$, and $\Sigma_{i j}=P_{i j \mid i-1}+\sigma_{\psi_{j}}^{2}$

Now, let $\boldsymbol{\theta}$ be the stacked vector of hyperparameters estimated, for instance by maximum likelihood. Then the bootstrap procedure is as follows:

Step 1: Build the standardized residuals:

$$
v_{i j}(\boldsymbol{\theta})=\Sigma_{i j}^{-1 / 2}(\boldsymbol{\theta}) \epsilon_{i j}(\boldsymbol{\theta})
$$

Step 2: Sample $\left\{v_{i j}(\boldsymbol{\theta})\right\}_{i=1}^{T}$ with replacement $T$ times. Denote $\left\{v_{i j}^{s}(\boldsymbol{\theta})\right\}_{i=1}^{T}$ the new series.

Step 3: Replace in $(21) \epsilon_{i j}$ by $\Sigma_{i j}^{1 / 2}(\boldsymbol{\theta}) v_{i j}^{s}(\boldsymbol{\theta})$ and compute a bootstrap series $z_{(i+1) j \mid i}^{s}(\boldsymbol{\theta})$. Replace in $(22) \epsilon_{i j}$ by $\Sigma_{i j}^{1 / 2}(\boldsymbol{\theta}) v_{i j}^{s}(\boldsymbol{\theta})$ and compute a bootstrap series $x_{i j}^{s}$ using $z_{(i+1) j \mid i}^{s}(\boldsymbol{\theta})$, the initial conditions being unchanged.

Step4: Re-estimate the model using $x_{i j}^{s}$. Compute $\widehat{\boldsymbol{\psi}}_{j}=\left(\widehat{\psi}_{1 j}, \widehat{\psi}_{2 j}, \ldots, \widehat{\psi}_{T j}\right)$ and $\widehat{\operatorname{Max}}_{j}=\max \left(\widehat{\psi}_{1 j}, \widehat{\psi}_{2 j}, \ldots, \widehat{\psi}_{T j}\right)$ and $\widehat{\operatorname{Min} \psi}_{j}=\min \left(\widehat{\psi}_{1 j}, \widehat{\psi}_{2 j}, \ldots, \widehat{\psi}_{T j}\right)$.

Step 5: Repeat the operation a large number of times, storing at each iteration $\widehat{\operatorname{Max}}_{j}$ and $\widehat{\operatorname{Min}}_{j}$.

The above procedure returns two series of bootstrap maximums and minimums. Following Bell and Hillmer (1984) and Harvey and Koopman (1992), 
it should be noted that even if $\widehat{\boldsymbol{\Psi}}$ is an estimator of the true measurement error, it won't inherit the iid property of $\boldsymbol{\Psi}$. In particular, it can be shown that $\widehat{\Psi}$ has a stationary ARMA structure. Nevertheless, following Rootzén (1986), for stationary ARMA processes, the Fisher-Tippett theorem still applies, and the correct law of the extremes is the Gumbel one. The scale and the location parameters of the law are then easily computed using a suitable procedure as maximum likelihood, probability-weighted moments, or simply moments ${ }^{7}$, and then the statistics (17) and (18).

If the data pass the above test, i.e. if the violations are caused by measurement errors, testing for weak separability is achieved by estimating the auxiliary model (9) with observed data by using an IV estimator ${ }^{8}$. A natural choice for the instruments being therefore given by the smoothed estimates of the state vector. This allows to take into account the amount of measurement error in the data. We next turn to a simulation study ${ }^{9}$.

\section{A small simulation study}

We now turn to a small Monte-Carlo simulation study in order to investigate the type I and II errors of the test under the stochastic case. Our general Data Generating Process (DGP) is as follows.

Step1. Given four monthly observed prices for durables, non-durables, services and food, and an income per capita for the United States over the period 1989:01-2004:10, we solve a representative consumer maximization program, whose utility is given by (24). Following Blackorby, Russel and Primont (1998) and Fleissig and Whitney (2003), note that this utility function is weakly separable over $\left(x_{i 3}^{*}, x_{i 4}^{*}\right)$ but not over $\left(x_{i 1}^{*}, x_{i 2}^{*}\right)$. It can thus be re-written as (25).

$$
\begin{gathered}
U_{i}=U\left(x_{i 1}^{*}, x_{i 2}^{*}, x_{i 3}^{*}, x_{i 4}^{*}\right)=x_{i 1}^{*(1 / 3)} x_{i 3}^{*(1 / 3)} x_{i 4}^{*(1 / 3)}+x_{i 2}^{*(1 / 2)} x_{i 3}^{*(1 / 4)} x_{i 4}^{*(1 / 4)} \\
U_{i}=V\left(x_{i 1}^{*}, x_{i 2}^{*}, f\left(x_{i 3}^{*}, x_{i 4}^{*}\right)\right)=x_{i 1}^{*(1 / 3)} v_{i}+x_{i 2}^{*(1 / 2)} v_{i}^{3 / 4}
\end{gathered}
$$


where:

$v_{i}=x_{i 3}^{*(1 / 3)} x_{i 4}^{*(1 / 3)}$

Step 2. Given model (11), we add to the computed quantities normally distributed iid terms. Three different measurement errors are used. Define the signal-to-noise ratio as $S N R_{j}=\sigma_{\xi_{j}}^{2} / \sigma_{\psi_{j}}^{2}$, where $\sigma_{\xi_{j}}^{2}$ is the variance of the residuals of the transition equation, and $\sigma_{\psi_{j}}^{2}$ is the variance of the measurement error for good $j, j=1, \ldots, k$ (see equation (20)). The signalto-noise ratio measures the degree of corruption of the data. Here, we use $S N R_{j}=0.5,1$ and 1.5. The smallest value corresponding to the largest measurement error (comparative to $\sigma_{\xi_{j}}^{2}$ ). Let $\mathbf{X}$ be the quantities with measurement error.

Step 3. We estimate the whole procedure type I error, that is the probability of incorrectly rejecting the weak separability. For this, we run GARP on $\left\{\left(\mathbf{x}_{i}, \mathbf{p}_{i}\right)\right\}_{i=1}^{T}$ and $\left\{\left(\mathbf{x}_{i}^{(1)}, \mathbf{p}_{i}^{(1)}\right)\right\}_{i=1}^{T}$ where here $\mathbf{x}_{i}^{(1)}=\left(x_{i 3}, x_{i 4}\right)$. If violations appear, we run (12) and test the significance of the violations by using (17) and (18). For this, we estimate the model (20) by maximum likelihood. We then bootstrap the Kalman filter (100 replications) to get two series of bootstrap extremes, estimate the parameters in (17) and (18), and test the significance. At a given threshold, if the data pass the test, we estimate (26) by using IV estimators, thus following Hsiao (1997).

$$
\log \left(\frac{\mathbf{p}_{\cdot 3}}{\mathbf{p}_{\cdot 4}}\right)=\alpha \log \left(\mathbf{x}_{\cdot 1}\right)+\beta \log \left(\mathbf{x}_{\cdot 2}\right)+\gamma \log \left(\mathbf{x}_{\cdot 3}\right)+\delta \log \left(\mathbf{x}_{\cdot 4}\right)+\mathbf{c}+\varepsilon(26)
$$

where $\mathbf{p}_{\cdot j}$ is the $j$ th column of $\mathbf{P}$, and $\mathbf{x}_{\cdot j}$ is the $j$ th column of $\mathbf{X}$. Two different kinds of instruments are used, i) The smoothed quantities computed thanks to the Kalman filter, ii) The smoothed quantities plus one lagged value for the observed quantities. Clearly, in (26) the separability of $\left(x_{i 3}, x_{i 4}\right)$ implies $\alpha=\beta=0$. Let $p_{w}$ be the $\mathrm{p}$-value of the restriction test (Wald). The type I error is therefore defined as the probability to have $\{\min ((17),(18))<$ threshold $\}$, or $\{\min ((17),(18)) \geq$ threshold and $p_{w}<$ threshold $\}$. 
Step 4. We estimate the whole procedure type II error, that is the probability of incorrectly accepting weak separability. For this, we run GARP on $\left\{\left(\mathbf{x}_{i}, \mathbf{p}_{i}\right)\right\}_{i=1}^{T}$ and $\left\{\left(\mathbf{x}_{i}^{(1)}, \mathbf{p}_{i}^{(1)}\right)\right\}_{i=1}^{T}$ where now $\mathbf{x}_{i}^{(1)}=\left(x_{i 1}, x_{i 2}\right)$. If violations appear, we run (12) and test the significance of the violations by using (17) and (18). As in step 3, we estimate the Kalman filter, forcing a local linear model. If the data pass the test, we estimate (27) by using IV estimators, using the same two sets of instrumental variables.

$$
\log \left(\frac{\mathbf{p}_{\cdot 1}}{\mathbf{p}_{\cdot 2}}\right)=\alpha \log \left(\mathbf{x}_{\cdot 1}\right)+\beta \log \left(\mathbf{x}_{\cdot 2}\right)+\gamma \log \left(\mathbf{x}_{\cdot 3}\right)+\delta \log (\mathbf{x} \cdot 4)+\mathbf{c}+\varepsilon(27)
$$

where $\mathbf{p}_{\cdot j}$ is the $j$ th column of $\mathbf{P}$, and $\mathbf{x}_{\cdot j}$ is the $j$ th column of $\mathbf{X}$. Clearly, the non-separability of $\left(x_{i 1}, x_{i 2}\right)$ implies $\gamma \neq 0$ and $\delta \neq 0$ in equation (27). Let $p_{w}$ be the p-value of the test. The type II error is defined as the probability to have $\left\{\min ((17),(18)) \geq\right.$ threshold and $p_{w} \geq$ threshold $\}$.

We repeat steps 2 to 41000 times. Tables (1) and (2) return the estimated type I and II errors with two different sets of instruments. Clearly, for the four considered thresholds, the type II error is set to zero, meaning that when data are measured with errors, the test perfectly recognizes non separability. Focusing on the type I error, at the standard $5 \%$ threshold and for small measurement error, it is about $0.055(0.059)$ and for the largest measurement error, 0.089 (0.105). The type I error thus remains small and within an acceptable range. Note that using only the smoothed estimates of the state vectors as instruments returns slightly better results.

\section{Conclusion and discussion}

In this paper, we have introduced a semi-nonparametric procedure to test for weak separability. With regard to the classical Varian's (1983) test, the one we have considered differs in two points. First, the only sufficient separability condition based on the Afriat inequalities is replaced by a necessary and sufficient one. This one uses the well known independence condition between the 
Table 1: Type I \& II errors for various signal-to-noise (SNR) ratios. Smoothed quantities as instruments.

\begin{tabular}{cccccccc}
\hline \hline & \multicolumn{3}{c}{ Type I error } & & \multicolumn{3}{c}{ Type II error } \\
\cline { 2 - 4 } \cline { 6 - 8 } Threshold & 0.5 & 1.0 & 1.5 & & 0.5 & 1.0 & 1.5 \\
\hline 0.01 & 0.025 & 0.024 & 0.016 & & 0.000 & 0.000 & 0.000 \\
0.05 & 0.089 & 0.084 & 0.055 & & 0.000 & 0.000 & 0.000 \\
0.10 & 0.151 & 0.132 & 0.114 & & 0.000 & 0.000 & 0.000 \\
0.15 & 0.216 & 0.178 & 0.161 & & 0.000 & 0.000 & 0.000 \\
\hline \hline
\end{tabular}

Table 2: Type I \& II errors for various signal-to-noise (SNR) ratios. Smoothed quantities and one lagged observed quantities as instruments.

\begin{tabular}{cccccccc}
\hline \hline & \multicolumn{3}{c}{ Type I error } & & \multicolumn{3}{c}{ Type II error } \\
\cline { 2 - 4 } \cline { 6 - 8 } Threshold & 0.5 & 1.0 & 1.5 & & 0.5 & 1.0 & 1.5 \\
\hline 0.01 & 0.032 & 0.025 & 0.021 & & 0.000 & 0.000 & 0.000 \\
0.05 & 0.105 & 0.079 & 0.059 & & 0.000 & 0.000 & 0.000 \\
0.01 & 0.189 & 0.137 & 0.115 & & 0.000 & 0.000 & 0.000 \\
0.15 & 0.257 & 0.188 & 0.183 & & 0.000 & 0.000 & 0.000 \\
\hline \hline
\end{tabular}

marginal rates of substitution between the goods inside the group and the goods outside. Second, the whole procedure is stochastic and deals with measurement error in the analysis. On simulated data, the test appears to be quite powerful, even for large random measurement errors.

At last, in this paper, to test the independence, we have used simple IV estimators. Alternativally, one may also consider using FM-GIVE or FM-IV estimators. This is let for future research.

\section{Notes}

\footnotetext{
${ }^{1}$ It could be nevertheless possible to combine the results of Fleissig and Whitney (2003) and Fleissig and Whitney (2005).

${ }^{2}$ Note that the matrix $\mathbf{Y}$ is not a $(T \times a(a-1))$ matrix since $\log \left(\frac{\mathbf{p}_{\cdot i}}{\mathbf{p}_{\cdot j}}\right)=-\log \left(\frac{\mathbf{p}_{\cdot j}}{\mathbf{p}_{\cdot i}}\right)$, and since such a matrix will not have full rank.
} 
${ }^{3}$ To avoid any confusions, note that equation (9) is only an independence test. Thus the estimated parameters will not be estimates of the parameters of the marginal rate of substitution. Exceptions are for instance for the cobb-douglas function.

${ }^{4}$ See Appendix 1 for computational details.

${ }^{5}$ On extreme values, see Embrecht, Klüppelberg and Mikosch (2003) and Guégan (2003).

${ }^{6}$ Note that such residuals are known as auxiliary residuals. They return an information about outliers in the series.

${ }^{7}$ See Johnson, Kotz, and Balakrishnan (1994).

${ }^{8}$ Interestingly, if the data are non stationary, a result of Hsiao (1997) states that classical IV estimators, as well as classical associated wald tests are still valid, without modifications. Kitamura and Phillips (1997) also suggest using FM-GIVE or FM-IV estimators. In empirical work, it may be useful to look at the two different estimators.

${ }^{9}$ In this section we have focused of the type I Gumbel distribution. An other solution, is a nonparametric one. By increasing the number of replications of the bootstrap procedure, it is possible to have an estimate of the two laws of the extremes. A standard quantile approach can then be used. This can nevertheless be quite computationally burdensome. 


\section{References}

[1] Barnett, W.A. and S.A. Choi (1989): A Monte Carlo study of tests of blockwise weak separability, Journal of Business \& Economic Statistics, Vol. 7, 363-377.

[2] Blackorby, C., Russell, R.R. and D. Primont (1998): Separability: A survey, in The Handbook of Utility Theory Vol. 1, S. Barbera, P. Hammond, and C. Seidl eds, Kluwer: Dordrecht, 49-92.

[3] Bell, W.R. and S.C. Hillmer (1984): Issues involved with the seasonal adjustment of economic time series, Journal of Business 83 Economic Statistics, Vol. 2, 291-320.

[4] de Peretti, P. (2005): Testing the significance of the departures from utility maximization, Macroeconomic Dynamics 9, 372-397.

[5] de Peretti, P. (2007): Testing the significance of the departures from weak separability, International Symposia in Economic Theory and Econometrics: Functional Structure Inference, William Barnett and Apostolos Serletis, Eds. Elsevier Press.

[6] D'agostino, R.B and M.A. Stephens (1986): Goodness-of-fit techniques, Statistics: Textbooks and Monographs, New York: Dekker, D'Agostino and Stephens, Eds.

[7] Deaton, A. and J. Muellbauer (1980): Economics and consumer behavior, Cambridge University Press.

[8] Durbin, J. and S.J. Koopman (2001: Time Series Analysis by State Space Methods. Oxford Statistical Science Series, Oxford University Press.

[9] Embrecht, P., Klüppelberg, C. and T. Mikosch (2003): Modelling extremal events, Springer-Verlag, New York.

[10] Fisher, D. and A.R. Fleissig (1997): Monetary aggregation and the demand for assets, Journal of Money, Credit and Banking 29,458-75. 
[11] Fleissig, A. R. and G.A. Whitney (2003): New PC-Based test for varian's weak separability conditions, Journal of Business 83 Economic Statistics, Vol. 21, No. 3, 133-144.

[12] Fleissig, A. R. and G.A. Whitney (2005): Testing for the significance of violations of Afriat's inequalities, Journal of Business 83 Economic Statistics, Vol. 23, No. 3, 355-362.

[13] Guégan, D. (2003): Introduction aux valeurs extrêmes et à ses applications, working paper, Ecole Normale Supérieure de Cachan.

[14] Harvey, A.C. (1989): Forecasting, structural time series models and the Kalman filter, Cambridge, U.K., Cambridge University Press.

[15] Harvey, A.C and S.J. Koopman (1992): Diagnostic checking of unobservedcomponents time series models, Journal of Business $\&$ Economic Statistics, Vol. 10, No.4, 377-389.

[16] Hosking, J.R.M. (1985): Maximum likelihood estimation of the parameter of the generalized extreme values distribution, Applied Statistics, 34, 301310.

[17] Hosking, J.R.M., Wallis, J.R and E.F. Wood (1985): Estimation of the generalized extreme-value distribution by the method of probability-weighted moments. Technometrics, 27, 251-261.

[18] Hsiao, C. (1997): Statistical properties of the two-stage least squares estimator under cointegration, The Review of Economic Studies, vol. 64, No. 3, 385-398.

[19] Johnson, N.L, Kotz, L. and N. Balakrishnan (1994): Continuous Univariate Distributions, Vol. I and II, 2nd. edition, Eds.: John Wiley and Sons.

[20] Jones, B., Elger, T., Edgerton, D. and D. Dutkowsky (2005): Toward a unified approach to testing for weak separability, Economic Bulletin, Vol. $3,1-7$. 
[21] Rootzén, H. (1986): Extreme value theory for moving average processes, The Annals of Probability, Vol. 14, No. 2, 612-652.

[22] Reinsel. G. (1997): Elements of multivariate time series, Springer-Verlag, New York.

[23] Kitamura, Y. and P.C. Phillips (1997), Fully modified IV,GIVE and GMM estimation with possibly non-stationary regressors and instruments, Journal of Econometrics 80, 85-123.

[24] Stoffer, D.S. and K. Wall, (1991): Bootstrapping state space models: Gaussian maximum likelihood estimation and the Kalman filter, Journal of the American Statistical Association 86, 1024-1033.

[25] Swofford, J.L. and G.A. Whitney (1987): Non-Parametric tests of utility maximization and weak separability for consumption, leisure, and money, Review of Economic Studies 69, 458-464.

[26] Swofford, J.L. and G.A. Whitney (1994): A revealed preference test for weakly separable utility maximization with incomplete adjustment, Journal of Econometrics 60, 235-49.

[27] Varian, H. (1982): The Nonparametric Approach to Demand Analysis, Econometrica 50, 945-973.

[28] Varian, H. (1983): Nonparametric Tests of Consumer Behavior, Review of Economic Studies 50, 99-110

[29] Varian, H. (1985): Non-Parametric Analysis of Optimizing Behavior With Measurement Error, Journal of Econometrics 30, 445-458. 
Solving the adjustment procedure (12)

Here we detail how the adjustment procedure (12) is solved, that is how we minimize the quadratic form subject to transitive constraints. What we want is to produce a set consistent with both:

i) The overall utility maximization program,

ii) The sub-utility maximization program, such that the distance with the observed data is minimal.

Following de Peretti $(2005,2007)$, define the following binary relation: $\mathbf{x}_{i} V R \mathbf{x}_{j}$ if $\mathbf{x}_{i} R \mathbf{x}_{j}$ and $\mathbf{x}_{j} P^{0} \mathbf{x}_{i}$ or there if is exists a sequence between $\mathbf{x}_{i}$ and $\mathbf{x}_{j}$ such that $\mathbf{x}_{i} R \mathbf{x}_{k}$ and $\mathbf{x}_{k} P^{0} \mathbf{x}_{i}, \mathbf{x}_{k} R \mathbf{x}_{l}$ and $\mathbf{x}_{l} P^{0} \mathbf{x}_{k}, \ldots, \mathbf{x}_{m} R \mathbf{x}_{j}$ and $\mathbf{x}_{j} P^{0} \mathbf{x}_{m}$. Also define $\mathbf{x}_{i} S R \mathbf{x}_{k}$ if $S(i)=S(j)$, where $S(i)=\left(\sum_{j=1}^{T} r_{i j}\right)-1, r_{i j}$ being the element at the $i t h$ row and $j t h$ column of the transitive closure matrix. Given the two above relations, the sequence we want to consider is as follows: i) First adjust the quantities to produce data consistent with the sub-utility, ii) second adjust the quantities to produce data consistent the overall utility, such that the data must still be consistent with the sub-utility.

Concerning the sub-utility adjustment program, for a set $D^{(1)}=\left\{\left(\mathbf{x}_{i}^{(1)}, \mathbf{p}_{i}^{(1)}\right)\right\}_{i=1}^{T}$ violating GARP the iterative adjustment procedure is as follows:

Step 1.1: Test $\left\{\mathbf{x}_{i}^{(1)}, \mathbf{p}_{i}^{(1)}\right\}_{i=1}^{T}$ for consistency with GARP, let $\mathbf{R}^{(1)}$ be the transitive closure matrix, and $r_{i j}^{(1)}$ be an element at the $i$ th row and $j$ th column. Define nvio as the number of violations: if: $\left\{\begin{array}{l}n v i o=0 \text { then stop the iterative procedure, } \\ \text { Otherwise go to step 1.2. }\end{array}\right.$

Step 2.1: Since $\mathbf{x}_{i}^{(1)} V R \mathbf{x}_{j}^{(1)}$ implies $\mathbf{x}_{i}^{(1)} S R \mathbf{x}_{j}^{(1)}$, build $B_{l} \operatorname{set}(\mathrm{s}), l=1, \ldots, n$ such that every couple $\left(\mathbf{x}_{i}^{(1)}, \mathbf{p}_{i}^{(1)}\right)$ and $\left(\mathbf{x}_{j}^{(1)}, \mathbf{p}_{j}^{(1)}\right)$ belonging to $B_{l}, \forall l \in\{1, \ldots, n\}$ satisfy $\mathbf{x}_{i}^{(1)} S R \mathbf{x}_{k}^{(1)}$. Search for the one, $B_{1}$, containing bundles violating GARP being all the same highest place in the preference chain such that $\left(\mathbf{x}_{i}^{(1)}, \mathbf{p}_{i}^{(1)}\right) \in B_{1}$ and $\left(\mathbf{x}_{j}^{(1)}, \mathbf{p}_{j}^{(1)}\right) \notin B_{1}: S(i)>S(j)$. Go to step 3.1.

Step 3.1: In the set $B_{1}$, search for all bundles related by $\mathbf{x}_{i}^{(1)} R \mathbf{x}_{j}^{(1)}$ and $\mathbf{x}_{j}^{(1)} P^{0} \mathbf{x}_{i}^{(1)}$, for the bundle that will be revealed preferred to the others. This is done 
by solving the program (28) and by selecting the bundle for which the objective function is minimal. Let $\left(\widehat{\mathbf{z}}_{i}^{(1)}, \mathbf{p}_{i}^{(1)}\right)$ be the bundle solution, where $\widehat{\mathbf{z}}_{i}^{(1)}=\left(\widehat{z}_{i 1}^{(1)}, \widehat{z}_{i 2}^{(1)}, \ldots, \widehat{z}_{i a}^{(1)}\right)$. Replace in $D^{(1)}\left(\mathbf{x}_{i}^{(1)}, \mathbf{p}_{i}^{(1)}\right)$ by $\left(\widehat{\mathbf{z}}_{i}^{(1)}, \mathbf{p}_{i}^{(1)}\right)$.

Go to step 1.1.

$$
o b j_{i}=\min _{z_{i j}^{(1)}} \sum_{j=1}^{a}\left(z_{i j}^{(1)}-x_{i j}^{(1)}\right)^{2}
$$

Subject to:

$$
\begin{aligned}
& \mathbf{p}_{i}^{(1)} \cdot \mathbf{x}_{i}^{(1)}=\mathbf{p}_{i}^{(1)} \cdot \mathbf{z}_{i}^{(1)} \text { and } \mathbf{p}_{j}^{(1)} \cdot \mathbf{x}_{j}^{(1)} \leq \mathbf{p}_{j}^{(1)} \cdot \mathbf{z}_{i}^{(1)}, \\
& \mathbf{p}_{m}^{(1)} \cdot \mathbf{x}_{m}^{(1)} \leq \mathbf{p}_{m}^{(1)} \cdot \mathbf{z}_{i}^{(1)} \text { for all } \mathbf{x}_{m}^{(1)} \text { such that } \mathbf{x}_{i}^{(1)} V R \mathbf{x}_{m}^{(1)}, m \neq j \\
& \mathbf{p}_{q}^{(1)} \cdot \mathbf{x}_{q}^{(1)} \leq \mathbf{p}_{q}^{(1)} \cdot \mathbf{z}_{i}^{(1)} \text { for all }\left(x_{q}^{(1)}, p_{q}^{(1)}\right) \notin B_{1} \text { such that } r_{i q}^{(1)}=1
\end{aligned}
$$

The above sequence returns a set $\widehat{D}^{(1)}=\left\{\left(\widehat{\mathbf{z}}_{i}^{(1)}, \mathbf{p}_{i}^{(1)}\right)\right\}_{i=1}^{T}$ consistent with the sub-utility maximization program, and a coherent transitive closure matrix $\widehat{\mathbf{R}}^{(1)}$. To simplify, define $\xi_{i}=\left(\boldsymbol{\xi}_{i 1}, \boldsymbol{\xi}_{i 2}, \ldots, \boldsymbol{\xi}_{i a}\right)=\widehat{\mathbf{z}}_{i}^{(1)}$, and then $\widehat{D}^{(1)}=\left\{\left(\boldsymbol{\xi}_{i}, \mathbf{p}_{i}^{(1)}\right)\right\}_{i=1}^{T}$. At last build the set $D=\left\{\left(\boldsymbol{\xi}_{i}, \mathbf{x}_{i}^{(2)}\right),\left(\mathbf{p}_{i}^{(1)}, \mathbf{p}_{i}^{(2)}\right)\right\}_{i=1}^{T}$.

The next step is now produce a set consistent with the overall utility, such that the data remain consistent with the sub-utility. This is done by using a similar iterative procedure, simply adding an additional constraint, such that the date must remain consistent with the transitive closure matrix $\widehat{\mathbf{R}}^{(1)}$. The procedure is as follows:

Step 2.1: Test $D=\left\{\left(\boldsymbol{\xi}_{i}, \mathbf{x}_{i}^{(2)}\right),\left(\mathbf{p}_{i}^{(1)}, \mathbf{p}_{i}^{(2)}\right)\right\}_{i=1}^{T}$ for consistency with GARP, let $\mathbf{R}$ be the transitive closure matrix, and $r_{i j}$ be an element at the $i$ th row and $j$ th column. Define nvio as the number of violations:

if: $\left\{\begin{array}{l}\text { nvio }=0 \text { then stop the iterative procedure, } \\ \text { Otherwise go to step 1.2. }\end{array}\right.$ 
Step 2.2: Since $\left(\boldsymbol{\xi}_{i}, \mathbf{x}_{i}^{(2)}\right) V R\left(\boldsymbol{\xi}_{j}^{(1)}, \mathbf{x}_{j}^{(2)}\right)$ implies $\left(\boldsymbol{\xi}_{i}^{(1)}, \mathbf{x}_{i}^{(2)}\right) S R\left(\boldsymbol{\xi}_{j}^{(1)}, \mathbf{x}_{j}^{(2)}\right)$, build $B_{l}$ set(s), $l=1, \ldots, n$ such that every couple $\left(\boldsymbol{\xi}_{i}^{(1)}, \mathbf{x}_{i}^{(2)}\right)$ and $\left(\boldsymbol{\xi}_{j}^{(1)}, \mathbf{x}_{j}^{(2)}\right)$ belonging to $B_{l}, \forall l \in\{1, \ldots, n\}$ satisfy $\left(\boldsymbol{\xi}_{i}^{(1)}, \mathbf{x}_{i}^{(2)}\right) S R\left(\boldsymbol{\xi}_{j}^{(1)}, \mathbf{x}_{j}^{(2)}\right)$. Search for the one, $B_{1}$, containing bundles violating GARP being all the same highest place in the preference chain such that $\left(\boldsymbol{\xi}_{i}^{(1)}, \mathbf{x}_{i}^{(2)}\right) \in B_{1}$ and $\left(\boldsymbol{\xi}_{j}^{(1)}, \mathbf{x}_{j}^{(2)}\right) \notin B_{1}: S(i)>S(j)$. Go to step 3.1.

Step 2.3: In the set $B_{1}$, search for all bundles related by $\left(\boldsymbol{\xi}_{i}^{(1)}, \mathbf{x}_{i}^{(2)}\right) R\left(\boldsymbol{\xi}_{j}^{(1)}, \mathbf{x}_{j}^{(2)}\right)$ and $\left(\boldsymbol{\xi}_{j}^{(1)}, \mathbf{x}_{j}^{(2)}\right) P^{0}\left(\boldsymbol{\xi}_{i}^{(1)}, \mathbf{x}_{i}^{(2)}\right)$, for the bundle that will be revealed preferred to the others. This is done by solving the program (29) and by selecting the bundle for which the objective function is minimal. Let $\left(\widehat{\mathbf{z}}_{i}^{(1)}, \widehat{\mathbf{z}}_{i}^{(2)}\right),\left(\mathbf{p}_{i}^{(1)}, \mathbf{p}_{i}^{(2)}\right)$ be the bundle solution. Replace in $D\left(\left(\boldsymbol{\xi}_{i}^{(1)}, \mathbf{x}_{i}^{(2)}\right),\left(\mathbf{p}_{i}^{(1)}, \mathbf{p}_{i}^{(2)}\right)\right)$ by $\left(\left(\widehat{\mathbf{z}}_{i}^{(1)}, \widehat{\mathbf{z}}_{i}^{(2)}\right),\left(\mathbf{p}_{i}^{(1)}, \mathbf{p}_{i}^{(2)}\right)\right)$. Go to step 3.1 .

$$
o b j_{i}=\min _{z_{i j}^{(1)}, z_{i j}^{(2)}}\left[\sum_{j=1}^{a}\left(z_{i j}^{(1)}-x_{i j}\right)^{2}+\sum_{j=a+1}^{k}\left(z_{i(j-a)}^{(2)}-x_{i j}\right)^{2}\right]
$$

Subject to:

$$
\begin{aligned}
\mathbf{p}_{i}^{(1)} \cdot \boldsymbol{\xi}_{i}+\mathbf{p}_{i}^{(2)} \cdot \mathbf{x}_{i}^{(2)} & =\mathbf{p}_{i}^{(1)} \cdot \mathbf{z}_{i}^{(1)}+\mathbf{p}_{i}^{(2)} \cdot \mathbf{z}_{i}^{(2)} \\
\mathbf{p}_{j}^{(1)} \cdot \boldsymbol{\xi}_{j}+\mathbf{p}_{j}^{(2)} \cdot \mathbf{x}_{j}^{(2)} & \leq \mathbf{p}_{j}^{(1)} \cdot \mathbf{z}_{i}^{(1)}+\mathbf{p}_{j}^{(2)} \cdot \mathbf{z}_{i}^{(2)} \\
\text { and } \mathbf{p}_{m}^{(1)} \cdot \boldsymbol{\xi}_{m}+\mathbf{p}_{m}^{(2)} \cdot \mathbf{x}_{m}^{(2)} & \leq \mathbf{p}_{m}^{(1)} \cdot \mathbf{z}_{i}^{(1)}+\mathbf{p}_{m}^{(2)} \cdot \mathbf{z}_{i}^{(2)} \\
\text { for all observations } & :\left(\boldsymbol{\xi}_{m}, \mathbf{x}_{m}^{(2)}\right) \text { satisfying }\left(\boldsymbol{\xi}_{i}, \mathbf{x}_{i}^{(2)}\right) V R\left(\boldsymbol{\xi}_{m}, \mathbf{x}_{m}^{(2)}\right), m \neq j
\end{aligned}
$$

where:

$$
\begin{aligned}
& z_{i}^{(1)}=\left(z_{i 1}^{(1)}, z_{i 2}^{(1)}, \ldots, z_{i a}^{(1)}\right) \\
& z_{i}^{(2)}=\left(z_{i 1}^{(1)}, z_{i 2}^{(1)}, \ldots, z_{i(k-a)}^{(1)}\right) \\
& \mathbf{p}_{q}^{(1)} \cdot \boldsymbol{\xi}_{q}+\mathbf{p}_{q}^{(2)} \cdot \mathbf{x}_{q}^{(2)} \leq \mathbf{p}_{q}^{(1)} \cdot \mathbf{z}_{i}^{(1)}+\mathbf{p}_{q}^{(2)} \cdot \mathbf{z}_{i}^{(2)} \text { for all }\left(\boldsymbol{\xi}_{q}^{(1)}, \mathbf{x}_{q}^{(2)}\right) \\
& \text { such that }: r_{i q}=1 .
\end{aligned}
$$




$$
\begin{aligned}
\mathbf{p}_{i}^{(1)} \cdot \boldsymbol{\xi}_{i}=\mathbf{p}_{i}^{(1)} \cdot \mathbf{z}_{i}^{(1)} \\
\mathbf{p}_{r}^{(1)} \cdot \boldsymbol{\xi}_{r} \leq \mathbf{p}_{r}^{(1)} \cdot \mathbf{z}_{i}^{(1)} \text { for all }\left(\boldsymbol{\xi}_{r}, \mathbf{p}_{r}^{(1)}\right) \\
\text { such that } \quad: \quad \widehat{r}_{i r}^{(1)}=1
\end{aligned}
$$

The above sequence returns a set $D=\left\{\left(\widehat{\mathbf{z}}_{i}^{(1)}, \widehat{\mathbf{z}}_{i}^{(2)}\right),\left(\mathbf{p}_{i}^{(1)}, \mathbf{p}_{i}^{(2)}\right)\right\}_{i=1}^{T}$ consistent with both the sub-utility maximization program and the overall utility maximization program, such that the L2 norm with the observed quantities is minimal. 